\title{
A GENERALIZATION OF QUASI-FROBENIUS RINGS
}

\section{HIROYUKI TACHIKAWA}

As a generalization of quasi-Frobenius rings, G. Azumaya [2] has investigated a ring with the property that every faithful left module is a generator ${ }^{1}$ in the category of left modules, and he has proved that such a ring is left self-injective and a direct sum of indecomposable left ideals having minimal left ideals.

In his proof, however, the existence of the faithful injective module plays an important role, while the injective module is not necessarily finitely generated, ${ }^{2}$ even if the ring is a left Artinian ring. Therefore, there naturally arises a problem: Is an Artinian ring quasi-Frobenius if every finitely generated faithful module is a generator?

The purpose of this paper is to give an affirmative answer to this problem as a direct consequence from a more general result which is rather similar to that of Azumaya stated above and related to perfect rings introduced by $\mathrm{H}$. Bass [3].

Throughout this paper we shall assume that the ring $R$ has identity element 1 and all modules over it are unital. For a subset $A$ of $R$ we shall denote

$$
r(A)=\{x \mid A x=0, x \in R\}, \quad l(A)=\{x \mid x A=0, x \in R\} .
$$

1. Preliminaries. Let $R$ be a ring. Let $M_{1}$ and $M_{2}$ be left $R$-modules and $T_{1}, T_{2}$ submodules of them respectively. Assume that there exists a left $R$-isomorphism $\theta: T_{1} \rightarrow T_{2}$ (onto). Let us denote by $L$ the factor module of $M_{1} \oplus M_{2}$ by the submodule consisting of all elements of form $\{m,-\theta(m)\}$ for $m \in T_{1}$. Then there are the canonical injections $\alpha$ and $\beta$ of $M_{1}$ and $M_{2}$ into $L$ respectively. In [12] we called $L$ the interlacing module of $M_{1}$ and $M_{2}$ by using $\theta$ as the lacing isomorphism and denote it by $\operatorname{Int}_{\theta}\left(M_{1}, M_{2}\right)$. In this paper, especially $\theta$ will be said to be maximal, ${ }^{3}$ if there is no isomorphism which is an extension of $\theta$.

In [3] H. Bass proved that for a right perfect ring $R$ the Jacobson radical $N$ of $R$ is right $T$-nilpotent and the residue class ring $\bar{R}=R / N$ is semisimple Artinian. Let $e$ be a primitive idempotent of $R$. The $R$-endomorphism ring of $R e$ is inverse-isomorphic to $e R e$, and $e R e$ is

Received by the editors January 20, 1967 and, in revised form, December 22, 1967.

${ }^{1}$ In his paper, G. Azumaya calls a generator a completely faithful module.

2 Cf. P. M. Cohn [5] and A. Rosenberg and D. Zelinsky [11].

3 The definition of "maximality" is different from that of [12]. 
completely primary (i.e. nonregular elements form the unique maximal ideal), because $e \mathrm{Ne}$ is nilideal. By the socle of a module we mean the sum of all simple submodules. It is known that every nonzero left $R$-module has nonzero socle if $R$ is a right perfect ring.

One of the following properties of modules is retained under the category-isomorphism: (a) simple, (b) finitely generated, (c) projective, (d) injective, (e) faithful, (f) generator. As we are concerned only with the above properties, we shall assume throughout that $R$ is isomorphic to its basic subring. Then, if $\left\{e_{\kappa}\right\}, \kappa=1,2, \cdots, n$, are mutually orthogonal primitive idempotents of $R$ such that $\sum_{k=1}^{n} e_{k}$ $=1, R e_{\kappa}$ is isomorphic to $R e_{\lambda}$ if and only if $\kappa=\lambda$.

2. Proof of the main theorem. Assume that $R$ is a right perfect ring. First we shall prove

Proposition 2.1. If every finitely generated faithful left $R$-module is a generator, then it follows that

(1) $e_{\lambda} r(N)$ is a simple right ideal of $R$.

(2) $r(N) e_{k}$ is a simple left ideal of $R$.

ProOF. Suppose $e_{\lambda} r(N)$ is nonzero right ideal. Consider the case (a): $e_{\lambda} r(N) e_{\kappa} \neq 0$ and $e_{\lambda} r(N) e_{\mu} \neq 0$ for $\kappa \neq \mu$. Take nonzero elements $c_{1}$ and $c_{2}$ such that $c_{1} \in e_{\lambda} r(N) e_{\kappa}$ and $c_{2} \in e_{\lambda} r(N) e_{\mu}$. Denote by $\theta$ the left $R$-isomorphism: $R e_{\lambda} c_{1} \rightarrow R e_{\lambda} c_{2}$, defined by $\theta\left(x e_{\lambda} c_{1}\right)=x e_{\lambda} c_{2}$ for $x e_{\lambda} c_{1}$ $\in R e_{\lambda} c_{1}$. Let us denote $\sum_{\rho \neq k, \rho \neq \mu} \oplus R e_{\rho} \oplus \operatorname{Int}_{\theta}\left(R e_{\kappa}, R e_{\mu}\right)$ by $L$. Then $L$ is a finitely generated, faithful left $R$-module, and hence by the assumption it follows that $L$ is a generator. Thus the trace ideal of $L$ must be $R$, and consequently there exist a family $\left\{x_{i}\right\}$ of elements of $L$ and a family $\left\{\phi_{i}\right\}$ of left $R$-homomorphisms $\phi_{i}: L \rightarrow R$ such that $\sum_{i=1}^{n} \phi_{i}\left(x_{i}\right)=1$. Since $L$ is a direct sum of $R e_{\rho}$ 's, $\rho \neq \kappa, \rho \neq \lambda$ and Int ${ }_{\theta}\left(R e_{\kappa}, R e_{\mu}\right), \phi_{i}$ can be expressed as follows: $\phi_{i}=\sum_{\rho \neq \kappa, \rho \neq \mu} \phi_{\rho, i}$ $+\phi_{(\kappa, \mu), i}$, where $\phi_{\rho, i}, \quad \phi_{(\kappa, \mu), i} \in \operatorname{Hom}_{R}(L, R), \phi_{\rho, i}\left(R e_{\rho^{\prime}}\right)=0$ for $\rho^{\prime} \neq \rho, \kappa, \mu, \phi_{\rho, i}\left(\operatorname{Int}_{\theta}\left(R e_{\kappa}, R e_{\mu}\right)\right)=0$, and $\phi_{(\kappa, \mu), i}\left(R e_{\rho}\right)=0$. On the other hand, to each $\phi_{\rho, i}$ and $\phi_{(x, \mu), i}$ there exist an element $e_{\rho} a_{\rho, i}$ of $R$ and a pair of elements $e_{\kappa} a_{\kappa, i}$ and $e_{\mu} a_{\mu, i}$ of $R$ such that $\phi_{\rho, i}\left(x_{\rho}\right)=x_{\rho} e_{\rho} a_{\rho, i}$ for all $x_{\rho} \in R e_{\rho}$ and $\phi_{(\kappa, \mu), i}\left(x_{\kappa} \alpha+x_{\mu} \beta\right)=x_{\kappa} e_{\kappa} a_{\kappa, i}+x_{\mu} e_{\mu} a_{\mu, i}$ for all $x_{\kappa} \in R e_{\kappa}$ and for all $x_{\mu} \in R e_{\mu}$. Here we notice that $c_{1} e_{k} a_{k, i}=c_{2} e_{\mu} a_{\mu, i}$, for $c_{1} e_{k} \alpha-c_{2} e_{\mu} \beta=0$ and $\phi_{(\kappa, \mu), i}\left(c_{1} e_{\kappa} \alpha-c_{2} e_{\mu} \beta\right)=0$. Then, putting $x_{i}=\sum_{\rho \neq \alpha, \rho \neq \mu} r_{\rho, i} e_{\rho}+r_{\alpha, i} e_{\alpha} \alpha$ $+r_{\mu, i} e_{\mu} \beta$, for $r_{\rho, i}, r_{k, i}, r_{\mu, i} \in R$, we have

$$
\sum_{i} \phi_{i}\left(x_{i}\right)=\sum_{\rho \neq \alpha, \rho \neq \mu}\left(\sum_{i} r_{\rho, i} e_{\rho} a_{\rho, i}\right)+\sum_{i} r_{k, i} e_{k} a_{\kappa, i}+\sum_{i} r_{\mu, i} e_{\mu} a_{\mu, i}=1 .
$$

It follows that 


$$
\sum_{\rho \neq k, \rho \neq \neq} \sum_{i} e_{k} r_{\rho, i} e_{\rho} a_{\rho, i} e_{k}+\sum_{i} e_{k} r_{x, i} e_{\alpha} a_{\alpha, i} e_{\alpha}+\sum_{i} e_{k} r_{\mu, i} e_{\mu} a_{\mu, i} e_{k}=e_{k}
$$

and hence $\sum_{i} e_{k} r_{k, i} e_{k} a_{k, i} e_{k} \equiv e_{k} \bmod e_{k} N^{2} e_{k}$. Since $e_{k} R e_{k}$ is completely primary, we can assume $e_{k} r_{k, 1} e_{k}$ and $e_{k} a_{k, 1} e_{k}$ are two regular elements of $e_{k} R e_{k}$.

Now, by a similar argument we can assume that $e_{\mu} r_{\mu, j} e_{\mu}$ and $e_{\mu} a_{\mu, j} e_{\mu}$ are two regular elements of $e_{\mu} R e_{\mu}$. Therefore $c_{1} e_{\kappa}=c_{2} e_{\mu} a_{\mu, 1}\left(e_{\kappa} a_{\kappa, 1} e_{\kappa}\right)^{-1}$ and $c_{1} e_{\kappa} a_{\kappa, j}\left(e_{\mu} a_{\mu, j} e_{\mu}\right)^{-1}=c_{2} e_{\mu}$ and consequently $c_{1} e_{\kappa}=c_{1} e_{\kappa} a_{\kappa, j}\left(e_{\mu} a_{\mu, j} e_{\mu}\right)^{-1}$ $\cdot e_{\mu} a_{\mu, 1}\left(e_{\kappa} a_{\kappa, 1} e_{\kappa}\right)^{-1}$. If we put $e_{\kappa} a_{\kappa, j} e_{\mu}\left(e_{\mu} a_{\mu, j} e_{\mu}\right)^{-1} e_{\mu} a_{\mu, 1} e_{\kappa}\left(e_{\kappa} a_{\kappa, 1} e_{\kappa}\right)^{-1}=n$, then $n \in e_{\kappa} N^{2} e_{\kappa}$ and $c_{1} e_{\kappa}(1-n)=0$. It follows that $c_{1} e_{\kappa}=0$, because $e_{\kappa} N e_{\kappa}$ is a nilideal. This is a contradiction.

Next we consider the case (b), $e_{\lambda} r(N) e_{\kappa}=e_{\lambda} r(N)$ and there exist two nonzero elements $c_{1}$ and $c_{2}$ of $e_{\lambda} r(N)$ such that $c_{1} \notin c_{2} R$ or $c_{2} \notin c_{1} R$. Since $r(N) e_{k}$ is completely reducible, the isomorphism $\theta: R e_{\lambda} c_{1} \rightarrow R e_{\lambda} c_{2}$ can be extended to an automorphism $\Theta$ of the socle $r(N) e_{\kappa}$ of $R e_{\kappa}$. Let $\bar{\Theta}$ be an extension of $\Theta$ and a subideal $S e_{\kappa}$ of $R e_{\kappa}$ the domain of $\bar{\Theta}$. Further, assume that $\bar{\Theta}$ is maximal. Then $S e_{\kappa} \neq R e_{\kappa}$, for otherwise $\bar{\Theta}$ should be obtained by the multiplication of a regular element $r$ of $e_{\kappa} R e_{\kappa}$ on the right hand and $c_{1} r=c_{2}$ and $c_{2} r^{-1}=c_{1}$, but this contradicts $c_{1} \notin c_{2} R$ or $c_{2} \notin c_{1} R$.

Let us denote by $M$ the left $R$-module $\sum_{\rho \neq k} \oplus R e_{\rho} \oplus \operatorname{Int} t_{\Theta}\left(R e_{\kappa}, R e_{\kappa}\right)$. Then, since $M$ is finitely generated, faithful, it follows that $M$ is a generator and the trace ideal of $M$ is $R$. There exist similarly as in case (a) a family $\left\{x_{i}\right\}$ of elements of $M$ and a family $\left\{\phi_{i}\right\}$ of left $R$ homomorphisms $\phi_{i}: M \rightarrow R$ such that $\sum_{i=1}^{n} \phi_{i}\left(x_{i}\right)=1$ and $\phi_{i}$ can be expressed as follows: $\phi_{i}=\sum_{\rho \neq k} \phi_{\rho, i}+\phi_{(\kappa, \kappa), i}$, where $\phi_{\rho, i}, \phi_{(\kappa, \kappa), i}$ $\in \operatorname{Hom}_{R}(M, R), \phi_{\rho, i}\left(\operatorname{Re}_{\rho^{\prime}}\right)=0$ for $\rho^{\prime} \neq \rho, \kappa, \phi_{\rho, i}\left(\operatorname{Int} \bar{t}_{\Theta}\left(R e_{\kappa}, \operatorname{Re}_{\kappa}\right)\right)=0$ and $\phi_{(x, x), i}\left(R e_{\rho}\right)=0$. To $\phi_{\rho, i}$ and $\phi_{(x, x), i}$ there exist an element $e_{\rho} a_{\rho, i}$ of $R$ and a pair of elements $e_{k} a_{k, i}$ and $e_{k} b_{k, i}$ of $R$ such that $\phi_{\rho, i}\left(x_{\rho}\right)=x_{\rho} e_{\rho} a_{\rho, i}$ for all $x_{\rho} \in R e_{\rho}$ and $\phi_{(\kappa, k), i}\left(x_{\kappa} \alpha+y_{k} \beta\right)=x_{k} e_{k} a_{\kappa, i}+y_{k} e_{k} b_{k, i}$ for all $x_{\kappa} \in R e_{\kappa}$ and for all $y_{k} \in R e_{\kappa}$. Here it is to be noted that $s e_{\kappa} a_{\kappa, i}=\bar{\Theta}\left(s e_{\kappa}\right) e_{\kappa} b_{k, i}$ for all $s e_{k} \in S e_{k}$. Then, putting $x=\sum_{\rho \neq k} r_{\rho, i} e_{\rho}+u_{k, i} e_{k} \alpha+v_{k, i} e_{k} \beta, r_{\rho, i}, u_{k, i}, v_{k, i}$ $\in R$, we have $\sum_{i} e_{\kappa} u_{\kappa, i} e_{k} a_{\kappa, i} e_{k}+\sum_{i} e_{k} v_{k, i} e_{k} b_{k, i} e_{k} \equiv e \bmod e_{k} N^{2} e_{\kappa}$ and at least an element of the set $\left\{e_{k} a_{k, i} e_{k}\right.$ and $\left.e_{k} b_{k, j} e_{k}, i, j=1,2, \cdots, n\right\}$ is regular in $e_{k} R e_{k}$. First suppose that $e_{k} b_{k, j} e_{k}$ be regular. Then $\bar{\Theta}\left(s e_{\kappa}\right)$ $=s e_{k} a_{k, j}\left(e_{k} b_{k, j} e_{k}\right)^{-1}$. Let us denote by $\Gamma$ the endomorphism of $R e_{k}$ which is obtained by the multiplication of $e_{k} a_{k, j} e_{k}\left(e_{k} b_{k, j} e_{k}\right)^{-1}$ on the right hand. Then $\Gamma$ is clearly an extension of $\bar{\Theta}$. This is a contradiction. Suppose, next, $e_{\kappa} a_{\kappa, i} e_{\kappa}$ be regular. Then $s e_{\kappa}=\bar{\Theta}\left(s e_{\kappa}\right) e_{\kappa} b_{k, i}\left(e_{k} a_{\kappa, i} e_{\kappa}\right)^{-1}$ and the endomorphism of $R e_{k}$ which is obtained by the multiplication of $e_{k} b_{k, i}\left(e_{k} a_{k, i} e_{k}\right)^{-1}$ is an extension of $\bar{\Theta}^{-1}$ and we arrive again at a 
similar contradiction. Thus, from the argument for the cases (a) and (b) we know that $e_{\lambda} r(N)$ is either simple or zero.

Now let us denote by $\Pi(\kappa)$ the set of primitive idempotents $e_{\rho}$ such that $e_{\rho} r(N) e_{\kappa} \neq 0$. Then, since $r(N) e_{\kappa} \neq 0$ for every $\kappa, \Pi(\kappa)$ is nonempty. If $\Pi(\kappa) \cap \Pi(\mu)$ is not empty for $\kappa \neq \mu$, then there exists a primitive idempotent $e_{\rho}$ such that $e_{\rho} r(N) e_{\kappa} \neq 0, e_{\rho} r(N) e_{\mu} \neq 0$. This contradicts the conclusion for the case (a). Thus $\Pi(\kappa)$ consists of only one primitive idempotent which we shall denote by $e_{\pi(\kappa)}$. The set $\left\{e_{\pi(\kappa)}\right\}$, for all $\kappa$, forms the set of all primitive idempotent $e_{\lambda}$ 's. Hence for every primitive idempotent $e_{\lambda}$ of $R$ there exists a primitive idempotent $e_{\kappa}$ such that $e_{\lambda} r(N) \cong \bar{e}_{\kappa} \bar{R}$, where $\bar{R}=R / N$. Therefore $e_{\lambda} r(N)$ is a nonzero ideal and (1) of this proposition holds good.

To prove (2), suppose $r(N) e_{\kappa}$ is not simple. Then, since $r(N) e_{\kappa}$ is completely reducible, there exists a nonzero endomorphism $\gamma$ such that $\operatorname{Ker} \gamma \neq 0$. Let us denote by $M$ the left $R$-module $R e_{\kappa} / \operatorname{Ker} \gamma$ and by $\bar{\gamma}$ the isomorphism of $r(N) e_{\kappa} / \operatorname{Ker} \gamma$ onto $\operatorname{Im} \gamma$ which is naturally induced by $\gamma$. Clearly, $\sum_{\rho \neq \kappa} \oplus \operatorname{Re}_{\rho} \oplus \operatorname{Int}_{\bar{\gamma}}\left(M, R e_{\kappa}\right)$ is finitely generated, faithful and hence a generator. Thus, similarly as in the preceding proof, there exist elements $e_{\rho} a_{\rho, i}, e_{\kappa} a_{k, i}, e_{k} b_{\kappa, i}$ such that (i) $v_{k, i} e_{k} a_{k, i}=0$ for all elements $v_{k, i} e_{k}$ of $\operatorname{Ker} \gamma$, (ii) $z_{\kappa, i} e_{\kappa} a_{\kappa, i}=\gamma\left(z_{k, i} e_{k}\right) e_{k} b_{k, i}$ for all elements $z_{k, i} e_{k}$ of $r(N) e_{k}$, and it holds $\sum_{\rho \neq k} \sum_{i} r_{\rho, i} e_{\rho} a_{\rho, i}$ $+\sum_{i} x_{k, i} e_{k} a_{k, i}+\sum_{i} y_{k, i} e_{k} b_{k, i}=1$, where $r_{\rho, i}, x_{k, i}, y_{k, i} \in R$. Hence $\sum_{i} e_{k} x_{k, i} e_{k} a_{k, i} e_{k}+\sum_{i} e_{k} y_{k, i} e_{k} b_{k, i} e_{k} \equiv e_{k} \bmod N^{2}$. However, by (i) it is known that $e_{\kappa} a_{\kappa, i} e_{\kappa}$ is a nonregular element of $e_{\kappa} R e_{\kappa}$. Hence, for some $i, e_{\kappa} b_{k, i} e_{\kappa}$ is a regular element of $e_{k} R e_{\kappa}$, and by (ii) $\gamma\left(z_{\kappa, i} e_{\kappa}\right)$ $=z_{\kappa, i} e_{k} a_{\kappa, i} e_{\kappa}\left(e_{\kappa} b_{k, i} e_{\kappa}\right)^{-1}$. On the other hand, by (1) of this proposition $r(N) \subseteq l(N)$ and $e_{\kappa} a_{\kappa, i} e_{\kappa} \in N$ and hence $\gamma\left(z_{\kappa, i} e_{\kappa}\right)=0$ for all $z_{\kappa, i} e_{\kappa} \in r(N) e_{\kappa}$. This contradicts that $\gamma$ is a nonzero endomorphism of $r(N) e_{k}$. (2) of this proposition follows. This completes the proof.

Following F. Kasch we shall say that $R$ is a left $S$-ring if $l(J) \neq 0$ for any right ideal $J$ with $J \neq R$.

COROLlaRY 2.2. Let $R$ be a right perfect ring. If every finitely generated, faithful left $R$-module is a generator, then $R$ is a left $S$-ring.

Proof. Let $J$ be a right ideal of $R$ such that $J \neq R$. Denote by $M$ the maximal right ideal containing $J$. Then for some $\kappa, R / M \cong \bar{e}_{\kappa} \bar{R}$, where $\bar{R}=R / N$. In the proof of (1) of Proposition 2.1, we have shown that there is an idempotent $e_{\lambda}$ such that $e_{\lambda} r(N) \cong \bar{e}_{\kappa} \bar{R}$, and hence there is a monomorphism of $R / M$ into $R$. This implies that there is a nonzero element $r$ of $R$ which annihilates $M$. Thus $0 \neq r \in l(M) \subseteq l(J)$.

Now we shall introduce a version of Azumaya's lemma [1, Lemma 1]. 
LеммA 2.3. Let $R$ be a ring (not necessarily right perfect ring) and $X$ a left $R$-module. If $X=M_{1} \oplus M_{2}$ and $X=N_{1} \oplus N_{2}$ are two direct sum decompositions of $X$, and the left $R$-endomorphism ring of $N_{1}$ is completely primary, then either $M_{1}$ or $M_{2}$ has a direct summand which is isomorphic to $N_{1}$.

Proof. For suitable idempotents $f_{1}, f_{2}, e_{1}$ and $e_{2}$ in the $R$-endomorphism ring $E$ of $X$ (considered as the right operator domain of $X$ ), we can assume that $M_{1}=X f_{1}, M_{2}=X f_{2}, N_{1}=X e_{1}, N_{2}=X e_{2}, f_{1}+f_{2}$ $=1$ and $e_{1}$ is a primitive idempotent of $E$. Since the $R$-endomorphism ring of $N_{1}$ is completely primary and $e_{1}=e_{1} f_{1} e_{1}+e_{1} f_{2} e_{1}$, either $e_{1} f_{1} e_{1}$ or $e_{1} f_{2} e_{1}$ must induce an automorphism of $N_{1}$, or what is the same, $N_{1}$ is mapped by $f_{1}$ or $f_{2}$ isomorphically upon $\tilde{N}_{1}$, and by $e_{1}, \tilde{N}_{1}$ is carried isomorphically onto $N_{1}$. Since $N_{2}$ is the kernel of $e_{1}$, the latter fact implies the following direct sum decomposition of $X: X=\tilde{N}_{1} \oplus N_{2}$. However, according as $e_{1} f_{1} e_{1}$ is regular or not, we can assume $\tilde{N}_{1}$ $=X e_{1} f_{1} \subset X f_{1}$ or $\tilde{N}_{1}=X e_{1} f_{2} \subset X f_{2}$. Hence the conclusion follows from the above decomposition of $X$.

Proposition 2.4. Let $R$ be a right perfect ring. If every finitely generated faithful left $R$-module is a generator, then $R$ is an injective left $R$-module.

Proof. Let $Q$ be the injective hull of any primitive ideal $R e_{\mathrm{k}}$. Assume that $R e_{\kappa}$ is contained properly in $Q$. Then there exists an element $u$ of $Q$ such that $R u \nsubseteq R e_{\kappa}$. Consider the left $R$-module $\sum_{\rho \neq k} \oplus R e_{\rho} \oplus R u+R e_{\kappa}$. It is obvious that this module is finitely generated, faithful, and hence a generator. It follows that $\left[\sum_{\rho \neq k} \oplus R e_{\rho} \oplus R u+R e_{k}\right]^{n}=\sum_{\rho \neq k} \oplus R e_{\rho} \oplus R e_{k} \oplus U$, where $U$ is a left $R$-module and $\left[\sum_{\rho \neq k} \oplus R e_{\rho} \oplus R u+R e_{\kappa}\right]^{n}$ is a direct sum of $n$-copies of $\sum_{\rho \neq k} \oplus R e_{\rho} \oplus R u+R e_{\kappa}$. Then, since $R e_{\rho} \not R e_{\kappa}$ and endomorphism ring of $R e_{\kappa}$ is completely primary, it follows by Lemma 2.3 that $R u+R e_{\kappa} \cong R e_{\kappa} \oplus U^{\prime}$ for a left $R$-module $U^{\prime}$. However, by (2) of Proposition 2.1 the socles of $R e_{\kappa}$ and $R u+R e_{\kappa}$ respectively are simple left $R$-modules. Therefore $U^{\prime}=0$ and $R u+R e_{\kappa} \cong R e_{\kappa}$. Hence $R e_{\kappa}$ is considered as a finitely generated, projective submodule of a projective left $R$-module $R u+R e_{\kappa}$. On the other hand, by Corollary $2.2 R$ is a left $S$-ring and by Bass's theorem ([3, Theorem 5.4], cf. also [3, Theorem 1]), $R u+R e_{\kappa}$ is a direct sum of $R e_{\kappa}$ and nonzero left $R$ module $V$. But this contradicts that the socles of $R u+R e_{\kappa}$ and $R e_{\kappa}$ are the same. Hence for every primitive idempotent $e_{k}, R e_{k}$ is injective.

Now we have the following main result.

THEOREM 2.5. Let $R$ be a right perfect ring. In order that every 
finitely generated, faithful left $R$-module be a generator it is necessary and sufficient that $R$ is left self-injective and a direct sum of primitive left ideals each of which contains a minimal left ideal.

Proof. The necessity follows from Proposition 2.4 and (2) of Proposition 2.1. On the other hand, it will be seen that the sufficiency is obtained by Azumaya's argument of [2, Theorem 6].

It is well known that a left Artinian ring is right perfect and a left Artinian ring is quasi-Frobenius if and only if it is injective as a left $R$-module. Thus we obtain

Theorem 2.6. Let $R$ be a left Artinian ring. In order that every finitely generated, faithful left $R$-module be a generator it is necessary and sufficient that $R$ is a quasi-Frobenius ring.

\section{REFERENCES}

1. G. Azumaya, Corrections and supplements to my paper concerning Krull-RemakSchmidt's theorem, Nagoya Math. J. 1 (1950), 117-124.

2. - Completely faithful modules and self-injective rings, Nagoya Math. J. 27 (1966), 697-708.

3. H. Bass, Finitistic dimension and a homological generalization of semi-primary rings, Trans. Amer. Math. Soc. 95 (1960), 466-488. 1962.

4. — The Morita theorems, Lecture notes, Univ. of Oregon, Eugene, Oregon,

5. P. M. Cohn, Quadratic extensions of skew fields, Proc. London Math. Soc. 11 (1961), 531-556.

6. Y. Kawada, On Köthe's problem concerning algebras for which every indecomposable module is cyclic. I, Sci. Rep. Tokyo Kyoiku Daigaku 7 (1962), 154-230.

7. K. Morita, Duality for modules and its applications to the theory of rings with minimum condition, Sci. Rep. Tokyo Kyoiku Daigaku 6 (1958), 83-142. 695.

8. —, On S-rings in the sense of F. Kasch, Nagoya Math. J. 27 (1966), 687-

9. T. Nakayama, On Frobeniusean algebras. I, Ann. of Math. 40 (1939), 611-633.

10. - On Frobeniusean algebras. II, Ann. of Math. 42 (1941), 1-21.

11. A. Rosenberg and D. Zelinsky, Finiteness of the injective hull, Math. Z. 70 (1958), 372-390.

12. H. Tachikawa, On rings for which every indecomposable right module has a unique maximal submodule, Math. Z. 71 (1959), 200-222.

Tokyo UnIVERSITY OF EDUCATION 